\title{
Early Detection of Dementia by Observing Change in the Driving Pattern of a Person using Smart Phone Sensors and DTW Algorithm
}

\author{
V. Subedha, PhD \\ Professor and Head, \\ Department of CSE, \\ Panimalar Institute of \\ Technology, \\ Chennai-600123.
}

\author{
T. Kalaichelvi, $\mathrm{PhD}$, \\ S. Hemalatha, PhD \\ Professor, \\ Department of CSE, Panimalar \\ Institute of Technology, \\ Chennai-600123.
}

\author{
J. Mithilaesh \\ Student, Fourth Year, \\ Department of CSE, Panimalar \\ Institute of Technology, \\ Chennai-600123.
}

\begin{abstract}
The word dementia describes a set of symptoms that could contain recollection defeat and difficulties alongside thinking, problem-solving or language. Dementia is provoked after the mind is broken by illnesses, such as Alzheimer's illness or a sequence of strokes. There are hundreds of requests obtainable to aid those recognized alongside dementia. But there are no apps or multimedia that can intimate a person whether he is possessing dementia or not. In established methods, a scope of examinations and diagnostic procedures are given to recognize dementia. These examinations for dementia are generally examinations of mental skills, blood examinations and mind scans. In this paper a novel method for noticing dementia is counseled by discerning recurrent adjustments in the steering outline of a person employing smartphone sensors and DTW algorithm, counseling possessing medication in advance beforehand the condition can become inferior in case the person is discovered to have dementia.
\end{abstract}

\section{Keywords}

Dementia, Alzheimer's, DTW (Dynamic Time warping), accelerometer, magnetometer, gyroscope, SMA (simple moving average).

\section{INTRODUCTION}

Dementia is not a specific disease. It's a finished word that describes an expansive scope of symptoms associated alongside a plummet in recollection or supplementary thinking skills harsh plenty to cut a person's skill to present everyday activities. As symptoms of Dementia can vary considerably, at least two of the pursuing core mental purposes have to be considerably impaired to be believed dementia:
1. Memory
2. Communication and language
3. Ability to focus and wage attention
4. Reasoning and judgment
5. Visual perception

Dementia is provoked by damage to mind cells. This damage interferes alongside the skill of mind cells to converse alongside every single other. After mind cells cannot converse normally, thinking, deeds and feelings can be affected. There is no solitary examination to ascertain if someone has dementia. Doctors recognize Alzheimer's and supplementary kinds of dementia established on a prudent health past, a physical examination, workshop examinations, and the characteristic adjustments in thinking, day-to-day purpose and deeds associated alongside every single type. Some of the symptoms shown by patients paining from dementia are

1. Memory defeat - exceptionally setbacks alongside recollection for present events, such as forgetting memos, recalling paths or terms, and asking inquiries repetitively.

2. Increasing difficulties alongside tasks and hobbies that need association and planning.

3. Becoming perplexed in unfamiliar environments.

4. Difficulty discovering the right words.

5. Difficulty alongside numbers and/or grasping money in shops.

6. Changes in personality and mood.

7. Depression.

\section{EXISTING SYSTEM}

At present there are android apps that aid in enhancing the mind purposes of a dementia patient by providing a little hobby like puzzles, asking inquiries, etc. There are additionally insufficient apps that assist dementia patients across disorientation by notifying them. But there no arrangement for noticing dementia preceding beforehand the person suffers critical condition.

\section{PROPOSED SYSTEM}

In the counseled arrangement we present a method to notice whether the person is possessing dementia by recognizing recurrent change in the steering outline of that person. This belief is established on the fact that Alzheimer's illness and supplementary dementias cause adjustments that alter a person's skill to drive a motor vehicle safely. A little of adjustments in the steering outline of dementia patients are

1. Traffic violations

2. Accidents

3. Getting lost

4. Misjudging distances

5. Forgetting the laws of the road

6. Slow reply times

7. Taking too long to grasp a destination.

8. Stopping the vehicle unexpectedly for no reason 


\section{LITERATURE SURVEY}

Various researchers have endeavored to monitor driver deeds employing both dedicated sensors used inside car, roadside and smartphone inbuilt sensors.

In [1] P. Singh et al. industrialized an android established request, this request accumulates data from accelerometers, GPS and additionally record sounds alongside the aid of microphone, and next data is joined and analyzed to notice rash steering patterns. The assorted outlines such as speed breaker, lane-change left/right, left/right coil, unexpected destroying, and unexpected quickening were analyzed and confirmed employing 'Ground Truth'. Correlation of audio and accelerometer data is completed to find new patterns.

In [2], Fazeen et al. have counseled an innovative request employing a mobile Smartphone that are consolidated inside an automobile to assess driver style. They have utilized the three-axis accelerometer of an Android-based smartphone to record and examine assorted driver behaviors and external road conditions that might potentially be hazardous to the condition of the driver. They have utilized $\mathrm{x}$-axis and $\mathrm{y}$-axis accelerometer data to compute the driver's manage manipulation of the vehicle as they drive, accelerate, and apply the brakes. Harmless quickening or deceleration not ever reaches a $\mathrm{g}$ force of extra than $\pm 0.3 \mathrm{~g}$, and unexpected quickening or deceleration way $\pm 0.5 \mathrm{~g}$. With this analogy, it is facile to quantify the difference amid harmless and unexpected quickening or deceleration. Harmless right/leftlane produce an average $g$-force of less than $\pm 0.1 \mathrm{~g}$ and unsafe or unexpected right/left lane produce a $\mathrm{g}$-force well over \pm 0.5 g. It was noted that the average period to finish a harmless lane change was $75 \%$ longer than an unexpected lane change. Phone arrangement locations in a vehicle was additionally noted and the loc. 1, the center console, provided the best comparative data alongside low engine feedback

In [3] Chigurupa et al. industrialized an android request that uses data from accelerometer sensor, GPS sensor and video recording is completed alongside the aid of camera to give locale to the driver. The feedback can be utilized to cognizant the driver and improve Performance. The scope of quickening or deceleration benefits is given for the harmless driving. Whenever the accelerometer benefits exceed the harmless limits it should be believed as an event. X-axis, direction-front and rear, steering pattern-Accelerating /Braking, Harmless $g$ worth $=-3$ to +3 . Y axis, direction-Left/right, steering patternTurning / Swerves / Lane Change, Harmless g worth $=-3$ to +3. Z-axis, direction-Up/down, driving Pattern-Bumps / Road Anomalies, Harmless g worth $=-8$ to- 11 .

In [4] Johnson et al. counseled a way for forecasting steering style. They categorized steering style into normal, hostile and extremely aggressive. They amass data from assorted sensors (accelerometer, gyroscope, magnetometer, GPS, video) and fused connected data into a solitary classifier established on Vibrant Period Warping (DTW) algorithm. Their arrangement is recognized as MIROAD: A Mobile-Sensor-Platform for Intelligent Credit of Hostile Driving, The arrangement can furnish audible feedback if a driver's style becomes hostile as well as the data managing up to a hostile event. They utilized iPhone 4, and noticed events like right turns, left turns, Uturns, hostile right, left, U-turns, hostile quickening, braking etc.

\section{SYSTEM MODULES}

The counseled arrangement is said to encompass of the pursuing modules
1. An intelligent phone alongside sensors (accelerometer, magnetometer, gyroscope).

2. Driving outline detection algorithm (DTW algorithm).

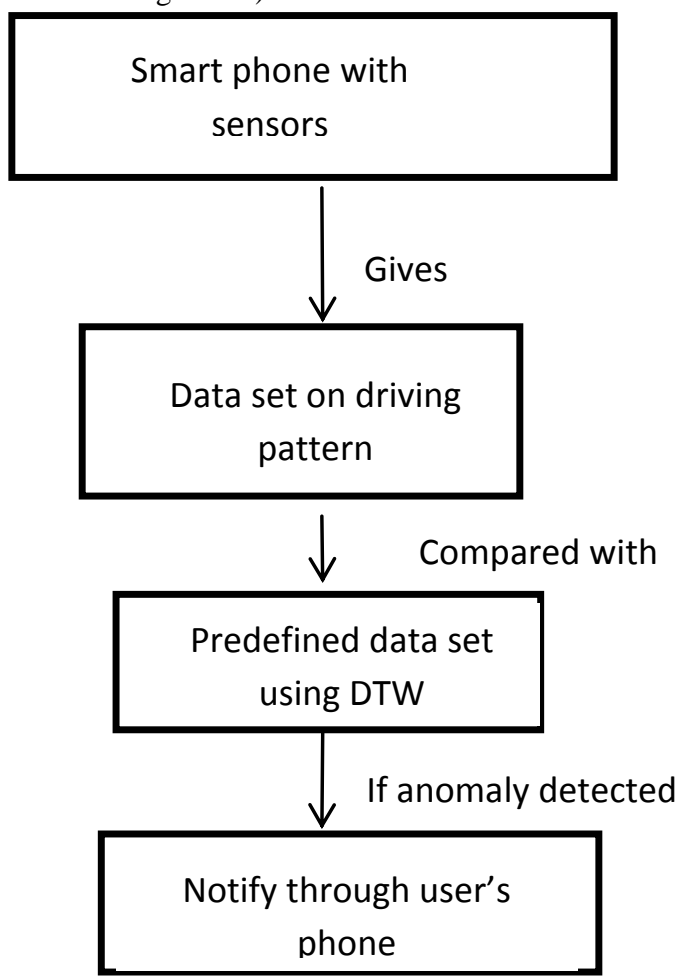

Fig 1.System architecture

\section{SMART PHONE WITH SENSORS}

The latest mobile phones are outfitted alongside countless functional inputs for scrutiny, encompassing, but not manipulated to:

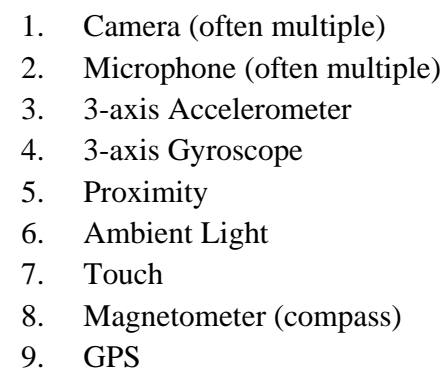

These mechanisms are influential, inexpensive and flexible scrutiny periods that make instrumenting a vehicle for data collection adjacent to the finished area as well as academia. In this arrangement our focus will be alongside the rear-facing camera, accelerometer, gyroscope and GPS (for event locale and speed only). For mechanism gesture, the axes of the phone are set up as shown in Fig. 2. With the climbing arm, we retain the mechanism rotated on its side and flush alongside the vehicle dashboard to stop it from advancing, and safeguarding the camera is unobstructed. In this arrangement we use the sensor-fusion output of accelerometer, gyroscope and magnetometer (compass) sensors to notice and categorize vehicle movement. The gyroscope signals are a clearer indication of vehicle coil movement, given that they compute rotation rate, and by employing the accelerometer and magnetometer in conjunction alongside the gyroscope, we can become an extra precise reading of mechanism attitude 
(orientation). The gyroscope measures rotation concerning itself, as the accelerometer adds correction alongside respect to gravity, and the magnetometer adds correction alongside respect to magnetic north.

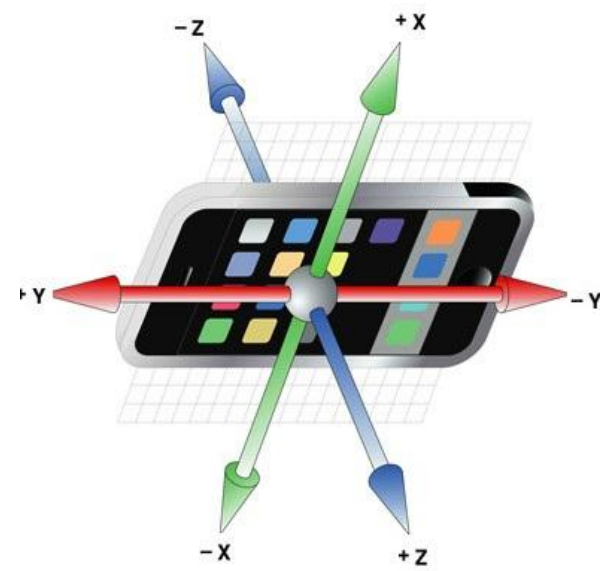

Fig. 2. The axes for accelerometer and gyroscope sensors

\section{DRIVING PATTERN DETECTION ALGORITHM}

The detection can be tear into two categories: lateral (turning) $\mathrm{T}$ and longitudinal $\mathrm{L}$ movements. These groups encompass mechanism gyroscope

values $\mathrm{G}=\{\mathrm{gx}, \mathrm{gy}, \mathrm{gz}\}$ in $\mathrm{rad} / \mathrm{s}$, mechanism accelerometer benefits $A=\{a x, a y, a z\}$ in $\mathrm{m} / \mathrm{s} 2$, and mechanism Euler slant rotation $\mathrm{E}=\{\mathrm{ex}, \mathrm{ey}, \mathrm{ez}\}$ in radians from a reference attitude $\mathrm{R}$.

$$
\begin{aligned}
\mathrm{T} & =\left\{\mathrm{g}_{\mathrm{x}}, \mathrm{a}_{\mathrm{y}}, \mathrm{e}_{\mathrm{x}}\right\} \\
\mathrm{L} & =\left\{\mathrm{g}_{\mathrm{y}}, \mathrm{a}_{\mathrm{z}}\right\}
\end{aligned}
$$

We selected to notice L separately, as it is simpler to threshold the $\mathrm{z}$-axis accelerometer worth independently for determining braking and acceleration. We trust that the set $\mathrm{T}=\{\mathrm{gx}, \mathrm{gy}, \mathrm{gz}$ \} is the best choice of signals for discriminating events alongside comparable movements. Here we use the DTW algorithm [4] on the three sets of signals remarked earlier: A, $\mathrm{G}$ and $\mathrm{T}$ to notice change in the steering pattern. The arrangement accumulates the gesture data from the accelerometer and gyroscope unceasingly at a rate of $25 \mathrm{~Hz}$ in order to notice specific maneuvers. The maneuvers of attention are hard left and right turns, swerves, and unexpected braking and quickening patterns. We early ascertain when a maneuver starts and ends employing endpoint detection. After we have a gesture representing a maneuver, we difference it to stored maneuvers (templates) to ascertain whether or not it matches an abnormal steering pattern. In order to notice after events started, we use an easy advancing average (SMA) [4] of the rotational power concerning the $\mathrm{x}$-axis for a window of size $\mathrm{k}$ from the present example i.

$$
S M A=\frac{g_{x}(i)^{2}+g_{x}(i-1)^{2}+\ldots+g_{x}(i-k-1)^{2}}{k}
$$

If SMA is larger than a higher threshold $\mathrm{tU}$ next $\mathrm{gx}(\mathrm{i}-\mathrm{k}-1)$ is the commencing of the event, and the consecutive benefits of gx are concatenated till SMA is less than a lower threshold tL. If the length of the event exceeds 15 seconds, the event is discarded. We selected the SMA of gx, because rotation stands out extra than quickening in all of our recorded events. We craft five templates for every single kind of event from all three sensor sets, totaling 40 recording maneuvers and 120 templates. We use the K-Nearest Neighbors (k-NN) association method alongside $\mathrm{k}=3$ to ascertain the kind of event. The five templates are industrialized employing a solitary vehicle and driver. Templates are recorded employing the alike endpoint detection method delineated above. For every single recorded event, three templates are saved simultaneously for the A, G and T sets of signals for precise comparison. The average steering events are seized from metropolis steering, as the abnormal steering events are seized in a controlled environment for safety. After trying to ascertain whether or not a steering event is usual or abnormal, the DTW algorithm finds the closest match amid the disparate styles of templates. The hostile templates encompass of elevated jerk movements and turns that cause defeat of traction. The kinds of events noticed by arrangement are:
1. Right turns (900)
2. Left turns $(900)$
3. U-turns (1800)
4. Irregular right turns (900)
5. Irregular left turns (900)
6. Irregular U-turns (1800)
7. Irregular acceleration
8. Irregular braking
9. Swerve right (Irregular lane change)
10. Swerve left (Irregular lane change)

By contrasting the recorded data set alongside the average template we notice the change in the steering outline of the arrangement and if recurrent change is noticed extra than 3-4 periods in a date next the person distrusted to have dementia and is intimated to have a consultation alongside a doctor across his intelligent phone

\section{ADVANTAGES OF THE PROPOSED SYSTEM}

Through the counseled arrangement one can understand he's possessing dementia beforehand the condition can become worse. This main diagnosis of dementia will enable you to:

1. Gain admission to data, resources and support

2. Demystify and destigmatise your condition

3. Maximize your quality of life

4. Benefit from treatments

5. Plan for the future

6. Explain to your relations, friends and associates what has modified in your life

\section{CONCLUSION}

Thus a novel method to notice dementia by discerning the adjustments in the steering outline of a person alongside the aid of smartphone sensors and a detection algorithm is counseled that might aid in notifying the person incase if he has dementia and counsel to go for medication so that we can circumvent the critical situations that should be provoked due to late diagnosis of the illness.

\section{REFERENCES}

[1] Singh, P., Juneja, N., Kapoor, S.: Using mobile phone sensors to detect driving behavior. In: Proceedings of the 3rd ACM Symposium on Computing for Development, ACM (2013).

[2] Fazeen, M., Gozick, B., Dantu, R., Bhukhiya, M., Gonzalez, M.C.: Safe Driving Using Mobile Phones. In 
International Journal of Computer Applications (0975 - 8887)

Volume 150 - No.11, September 2016

IEEE Transactions on Intelligent Transportation Systems (2012).

[3] Chigurupa, S., Polavarap, S., Kancherla,Y., Nikhath, K.A.:Integrated Computing System for measuring Driver Safety Index. In: International Journal of Emerging
Technology and Advanced Engineering, ISSN 22502459, Volume 2 (2012)

[4] Johnson, D.A., Trivedi, M.M.:Driving Style Recognition using a smartphone as a sensor platform. In: IEEE 14th International Conference on Intelligent Transportation system, October (2011) 